\title{
HOW TO DO IT:
}

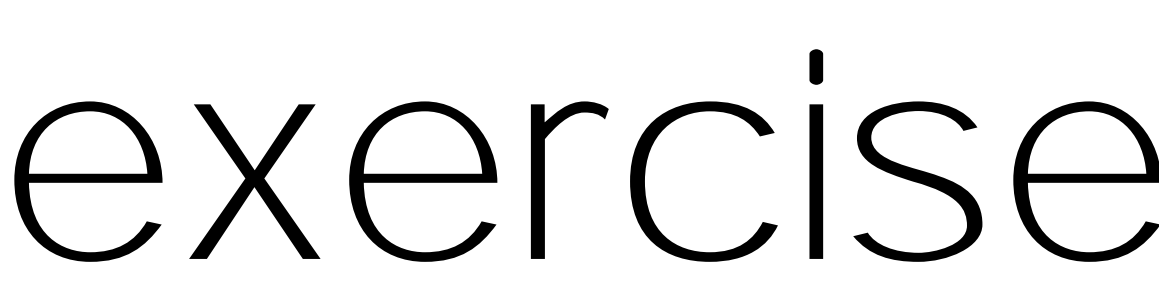
. 

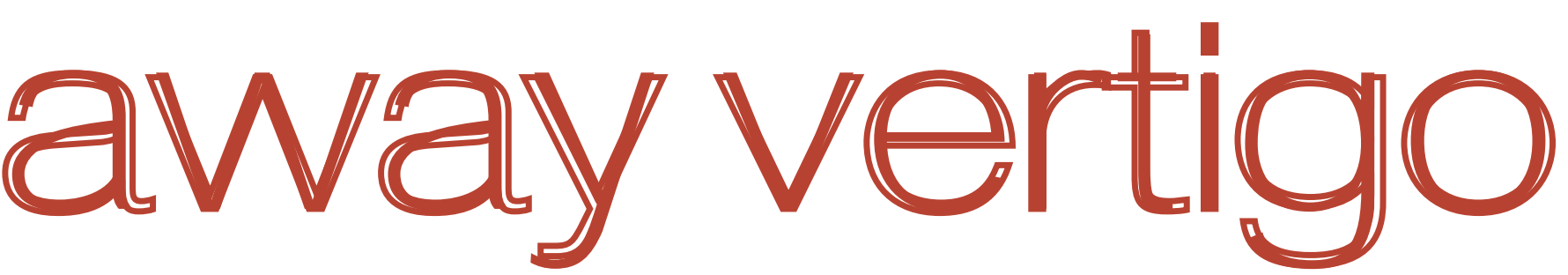

\section{Thomas B randt}

Klinikum Grosshadern, Ludwig-Maximilians University, M unich, Germany. E-mail: tbrandt@brain.nefo.med.unimuenchen.de

\section{INTRODUCTION}

Vertigo generally has a good prognosis because:

- many forms of vertigo have a benign cause and are characterized by spontaneous recovery of vestibular function, or central compensation of a peripheral vestibular tone imbalance; and;

- most forms of vertigo can be effectively relieved by pharmacological treatment, surgery, psychotherapy, or physical therapy.

Physical therapies include exercises for vestibular rehabilitation, promotion of central compensation of an acute vestibular loss, habituation for preventing motion sickness, and improvement of balance skills in the elderly. Themostimportantexercisesarethosethatfall within the scope of this article: deliberate manoeuvresfor positional vertigo (Brandt 1999).

\section{BENIG N PAROXYSMAL}

\section{POSITIONAL VERTIGO}

Benign paroxysmal positional vertigo (BPPV) wasinitially defined by Bárány (1921). It isthe most common cause of vertigo, particularly in the elderly. By the age of 70 , about $30 \%$ of all elderly people have experienced BPPV at least once. This condition is characterized by brief attacks of rotatory vertigo and concomitant positional rotatory-linear nystagmus, provoked by rapid changes in head position relative to gravity.

Patients with typical BPPV report attacks of rotatory vertigo, postural imbalance, and sometimes nausea precipitated by the following manoeuvres:
- sitting up from a supine position (particularly after awaking in the morning);

- when first lying down in bed;

- turning over in bed from one side to the other;

- extending theneck (head) to look up or get something from above;

- flexing theneck (head) when bending over. BPPV in the supine position is very disturbing. It makes patients afraid of falling backwards, an almost unique complaint. In the upright position, vertigo attacks produced by changes in head position are incapacitating. They can also be dangerous, for example when a sufferer looks up at the ceiling while standing on a ladder.

BPPV is a mechanical disorder of theinner ear in which the precipitating positioning of the head causes abnormal stimulation (Fig. 1), usually of the posterior semicircular canal ( $p$-BPPV) of theundermostear, lessfrequently of the horizontal (h-BPPV) and the anterior (a-BPPV) semicircular canal. Theobservation of positional nystagmus provides the definitivediagnostic criteriafor typical p-BPPV. They include:

- latency: vertigo and nystagmus begin one or moresecondsafter thehead istilted toward the affected ear and increase in severity to a maximum;

- duration lessthan $40 \mathrm{~s}$ : nystagmus gradually lessens after 10-40 s and ultimately abates, even when the precipitating head position is maintained;

- linear-rotatory nystagmus: the nystagmus is best seen if thepatient wearsFrenzel'sglass- 


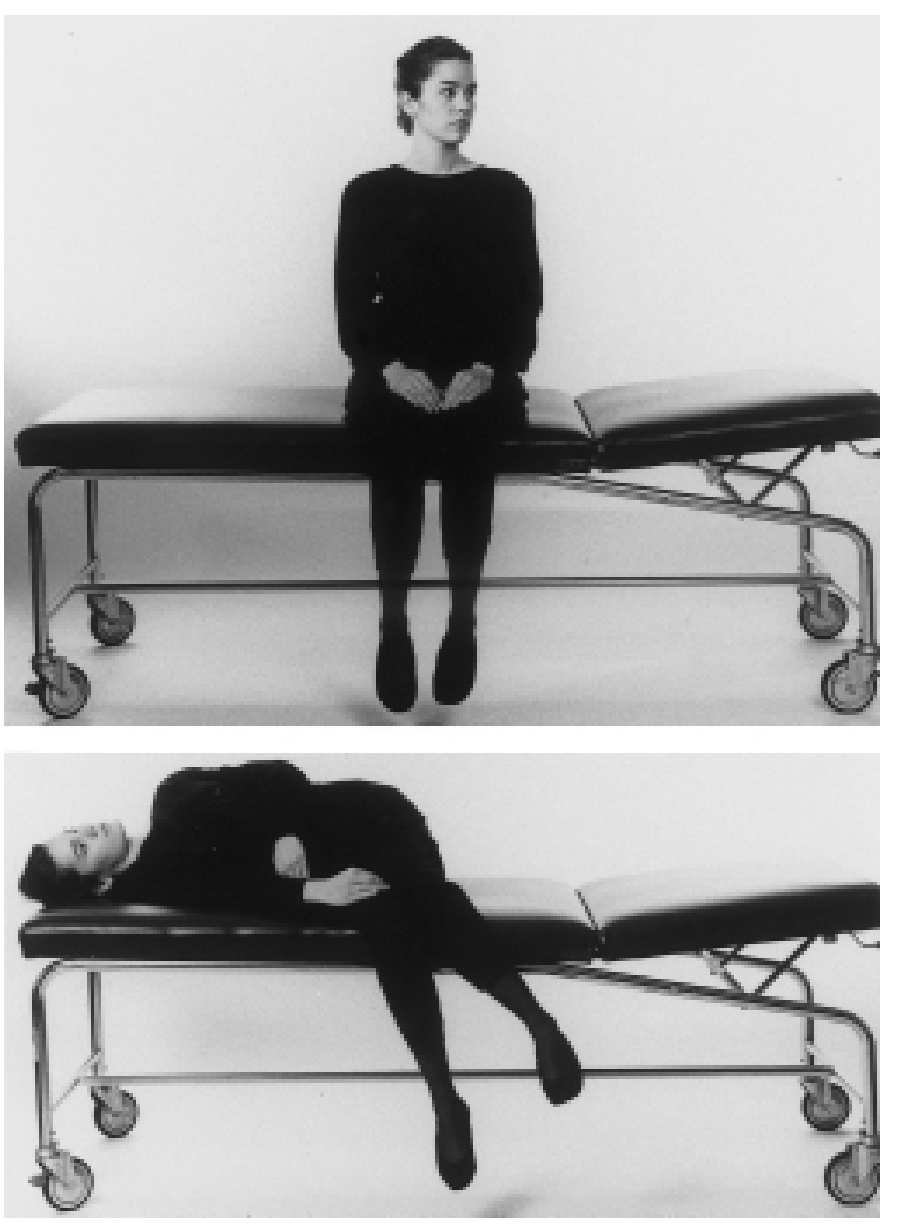

Fig. 1 Positional exercises for effective physical therapy for BPPV as proposed by Brandt \& Daroff (1980). Patients are instructed to sit and then to move rapidly into the challenging position, to remain in that position for at least $30 \mathrm{~s}$, and then to sit up for $30 \mathrm{~s}$ before assuming the opposite head-down position for 30

s. These exercises are repeated serially $5-10$ times a day.

es (for example, lenses $+16 \mathrm{dpt}$ ), which prevent suppression by fixation. The nystagmus is linear-rotatory, with the fast phase beating toward the undermost ear or upward when gaze is directed to the uppermost ear;

- reversal: when the patient returns to the seated position, the vertigo and nystagmus may re-occur, but lessviolently and in theoppositedirection;

- fatigability: constant repetition of the manoeuvre will result in ever-lessening symptoms.

These five criteria are crucial for further discussion of the confusing literature on the mechanism of BPPV. They providethemajor argumentsto proveor disproveany hypothetical explanation of cupulolithiasis or canalolithiasis as the causative factor.

\section{FLOATING OTOCONIA IN THE LAB YR INTH, C ANALOLITHIASIS \\ It is now generally accepted that debris floats}

freely within theendolymph of the canal ('canalolithiasis'). The debris - possibly particles detached from the otoliths- congeals to form a free-floating clot or plug. Because the clot is heavier than the endolymph, it will always gravitate to the most dependent part of the canal during changes in head position that alter the angle of the cupular planerelative to gravity. Analogous to a plunger, the clot induces bidirectional (push or pull) forces on the cupula, thereby triggering the BPPV attack. Canalolithiasis explains all the features of BPPV (Brandt \& Steddin 1993; Fig. 2):

- a latency of a few seconds (time needed for the clot-induced flow mechanism to develop by gravitational force);

- the ineffectiveness of a very slow positioning manoeuvre (then the clot would slowly gravitate along the undermost wall of the canal without affecting the cupula);

- theshort duration of thepositional vertigo/ nystagmus (cupula deflection due to elastic restoring force ends when the heavy clot reaches its lowest position in the canal with respect to the earth's surface);

- the fatigability with repetitive provocation (explained by dispersion of single particles from the clot, which decreases the plunger effect);

- the reactivation of the vertigo after prolonged bedrest (the result of a new clot being formed by the particles);

- the direction of the nystagmus during the positioning manoeuvres (explained below).

\section{PRECIPITATING POSITIONING MANOEUVRES CAUSE BOTH}

\section{THE MALAISE AND ITS RELIEF}

The positional exercises proposed in 1980 (Brandt \& Daroff 1980) were the first effective physical therapy (Fig. 1). They were a sequence of rapid lateral head/trunk tilts, repeated serially to promote dispersion of the debris toward the utricular cavity. We instructed the patients to sit; to then moverapidly into the challenging position to induce the correct plane-specific stimulation of the posterior semicircular canal; to remain in that position until the evoked vertigo subsided, or for at least $30 \mathrm{~s}$; and then to sit up for 30 $s$ before assuming the opposite head-down position for an additional $30 \mathrm{~s}$. Troost $\&$ Pat- 

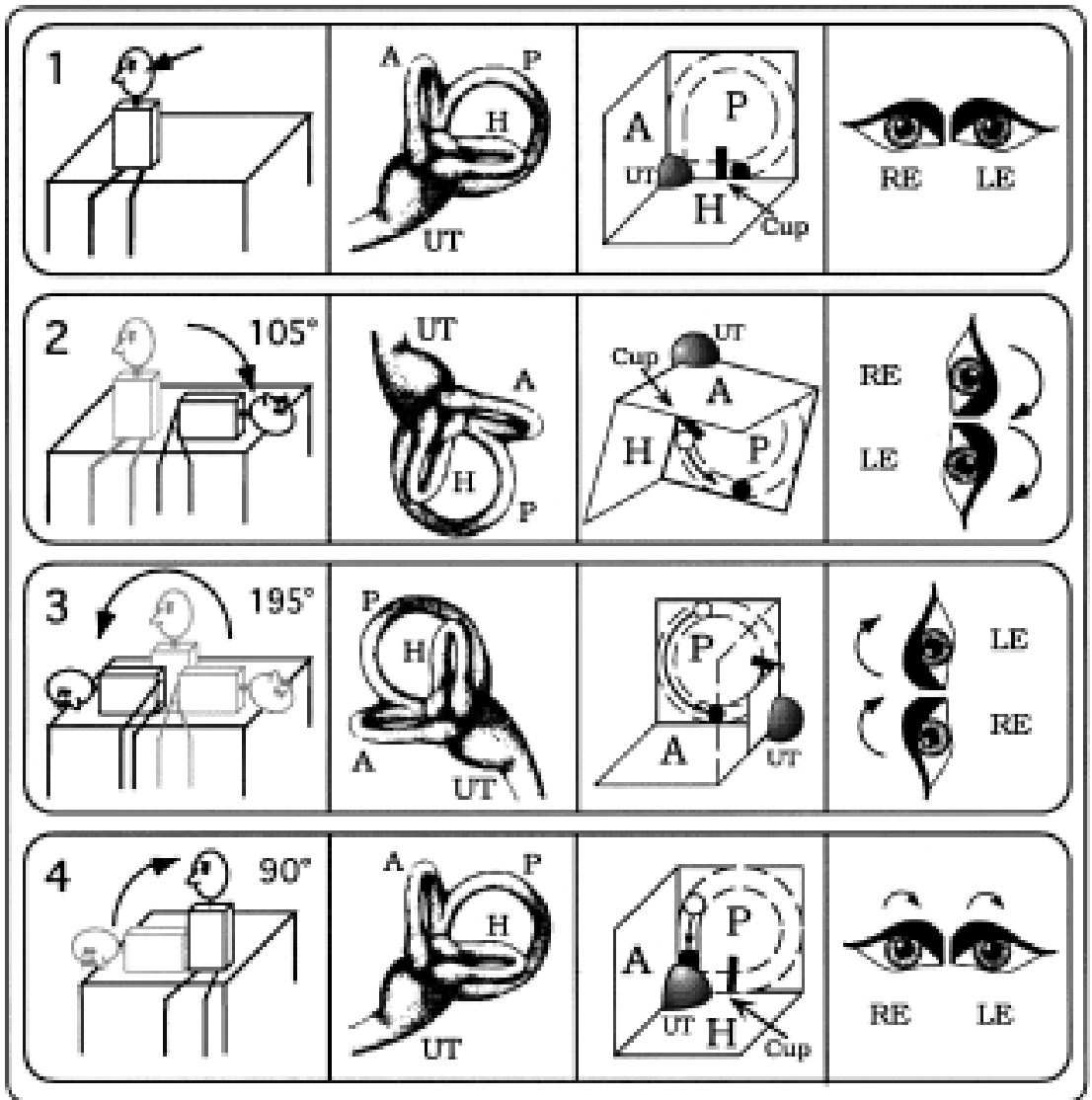

Complete recovery

after a single

\section{manoevre is achieved}

\section{in $50-70 \%$ of cases.}

Fig. 2 Schematic drawing of the Semont liberatory manouevre in a patient with typical (posterior canal) BPPV of the left ear. Boxes from left to right: position of body and head, position of labyrinth in space, position and movement of the clot in the posterior canal and resulting cupula deflection, and direction of the rotatory nystagmus. The clot is depicted as an open circle within the canal; a black circle represents the final resting position of the clot. Panel 1: In the sitting position, the head is turned horizontally $45^{\circ}$ to the unaffected ear. The clot, which is heavier than endolymph, settles at the base of the left posterior semicircular canal. Panel 2: The patient is tilted approximately $105^{\circ}$ toward the left (affected) ear. The change in head position, relative to gravity, causes the clot to gravitate to the lowermost part of the canal and the cupula to deflect downward, inducing BPPV with rotatory nystagmus beating toward the undermost ear. The patient maintains this position for 3 min. Panel 3: The patient is turned approximately $195^{\circ}$ with the nose down, causing the clot to move toward the exit of the canal. The endolymphatic flow again de-

flects the cupula such that the nystagmus beats toward the left ear, now uppermost. The patient remains in this position for 3 min. Panel 4: The patient is slowly moved to the sitting position; this causes the clot to enter the utricular cavity. Abbreviations: $A$, anterior semicircular canals; $P$, posterior semicircular canals; $\mathrm{H}$, horizontal semicircular canals; Cup, cupula; UT, utricular cavity; RE, right eye; LE, left eye. (From Brandt et al. 1994.)

ficacy of the therapies according to anatomic and physical principles.

Figure 2 illustrates the Semont manoeuvre in a patient with typical (posterior canal) left-sided BPPV. The clot causes no deflection of the cupula in the upright position. When the patient isquickly tilted toward the affected left ear with $45^{\circ}$ head rotation to the right (moving the left posterior canal to a plane corresponding to theplane of thehead tilt), theclot gravitatestoward thelower part of the canal, causing the cupula to deflect downward (ampullofugal), so triggering atypical BPPV attack.

If thepatient isswung toward theoppositeright sidewith thenosedown, the clot will gravitate downward, causing stimulation of the posterior canal of the affected left ear (now uppermost). If no vertigo and nystagmus are elicited, we gently shakethepatient'shead in thisposition; thissometimesseemsto facilitate settlement of the clot. The patient is then slowly moved to the upright posi- 


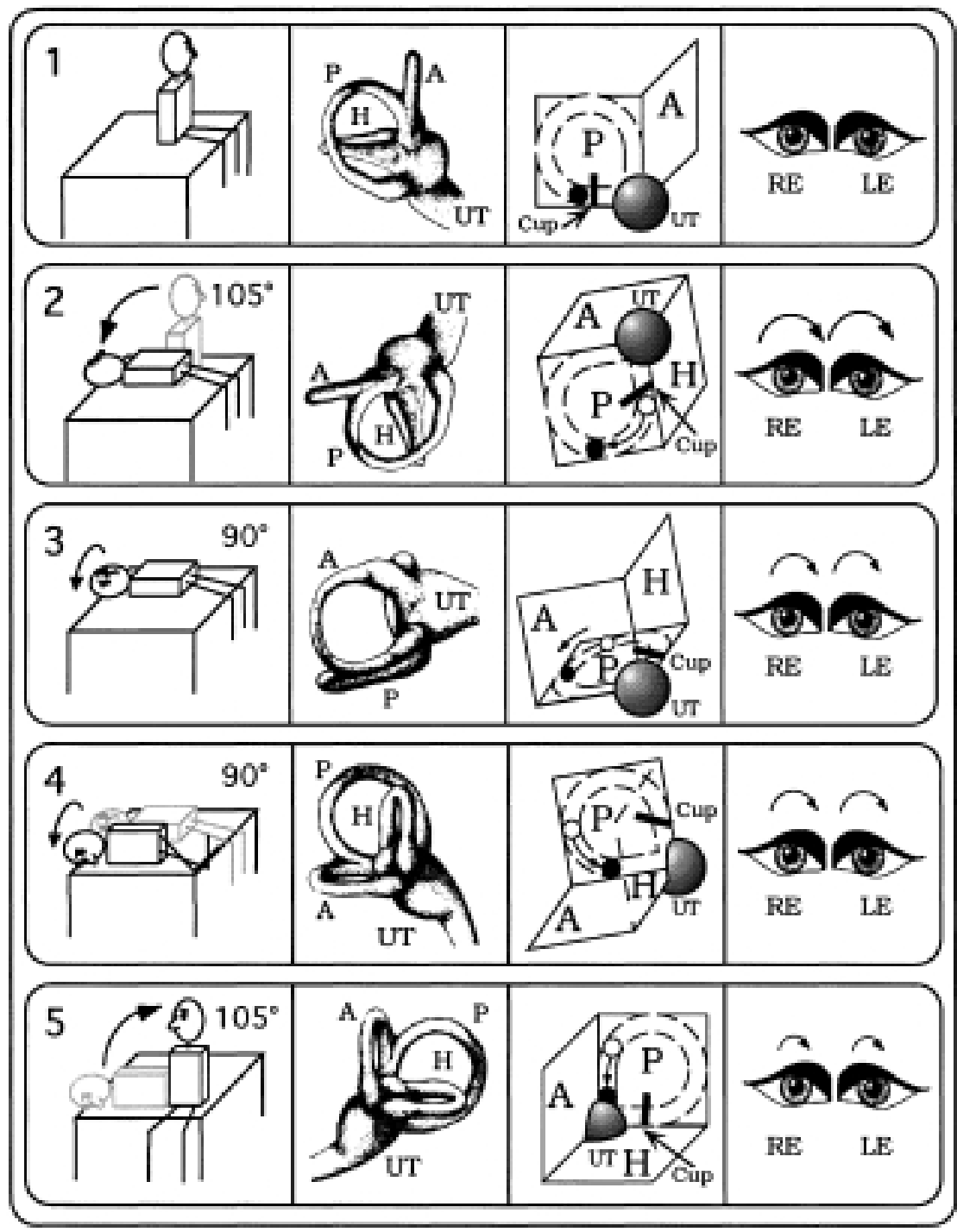

Fig. 3 Schematic drawing of the modified Epley liberatory manoeuvre. Patient characteristics and abbreviations are as in Fig. 2. Panel 1: In the sitting position, the head is turned horizontally $45^{\circ}$ to the affected (left) ear. Panel 2 : The patient is tilted approximately $105^{\circ}$ backward into a slight head-hanging position, causing the clot to move in the canal, deflecting the cupula downward, and inducing the BPPV attack. The patient remains in this position for 3 min. Panel 3: The head is turned $90^{\circ}$ to the unaffected ear, now undermost. Panel 4 : the head and trunk continue turning another $90^{\circ}$ to the right, causing the clot to move toward the exit of the canal. The patient remains in this position for 3 min The positional nystagmus beating toward the affected (uppermost) ear in positions 3 and 4 indicates effective therapy. Panel 5 : The patient is moved into the sitting position. (From Brandt et al. 1994.) tion; theclot will gravitatedownward through thecommon crus of theposterior and anterior canals and enter the utricular cavity, where it becomesharmless. Wesharetheexperience of others (Serafini et al. 1996) that completerecovery after a single manoeuvre is achieved in about $50-70 \%$ of cases. Semont et al. (1988) recommended having the patient maintain the upright position for $48 \mathrm{~h}$ following the treatment, but we have not found this necessary.

Figure 3 illustrates the Epley manoeuvre (1992) as modified by Herdman et al. (1993) and others(Harvey etal. 1994) in a patient with typical (posterior canal) left-sided BPPV. The clot causes no deflection of the cupula in the uprightposition with thehead turned horizontally $45^{\circ}$ to the affected ear. When the patient is quickly tilted backward into a slight headhanging position, theclot gravitates downward in the posterior canal, deflecting the cupula downward and inducing a BPPV attack. Rotation of the head and trunk toward the unaffected right ear causes further movement of the clot downward (ampullofugal) toward the exit of thecanal, resulting in positional vertigo and nystagmus toward the affected (now uppermost) ear. The final uprighting of the patient causestheclot to enter theutricular cavity, whereit becomes harmless.

Following effective physical liberation, approximately $50 \%$ of patients (Baloh et al. 1987) experiencea recurrence of attacks; $10 \%$ to $20 \%$ occur in thefirst two weeks (H erdman et al. 1993). The recurrences may be due to reentry of the debris into the posterior canal from the utricular cavity and should betreated with thesamemanoeuvrethat induced resolution of theinitial episode.

The process illustrated in Figs 2 and 3 explains the seemingly paradoxical observation (Semont et al. 1988) that the final liberatory positioning with the affected ear uppermost (Fig. 2, panel 3; Fig. 3, panel 4) induces nystagmusthat beatstoward that ear (Brandt \& Steddin 1993). As described above, cupulolithiasis predicts an ampullopetal deflection of thecupula that would cause nystagmus to beat toward the undermost ear, whereas in canalolithiasis, theclot-induced endolymphatic flow causes ampullofugal deflection of the cupula and nystagmus beating to the uppermost ear. 


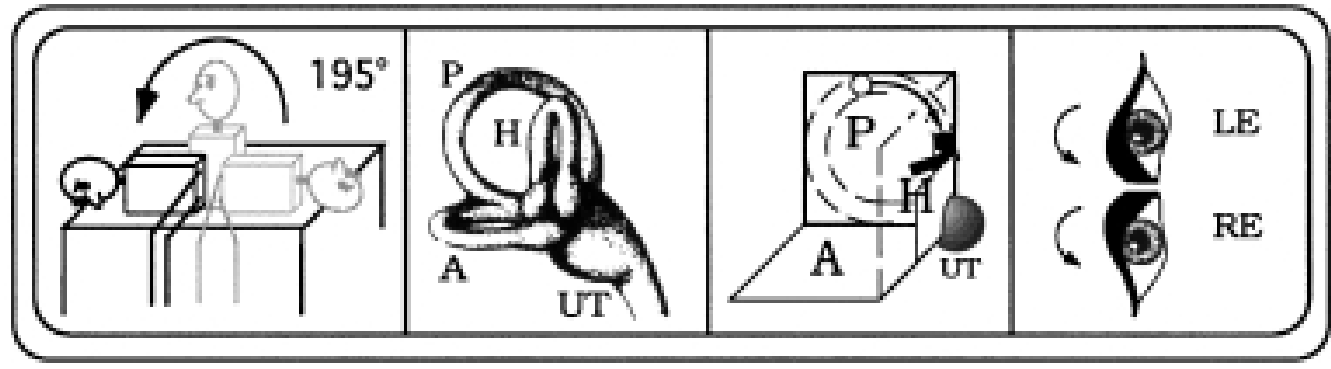

M oreover, theupward direction of thenystagmusinduced by thefinal positioningsisaclinically relevant observation in that it provides reasonablecertainty that theclothas exited the canal (or will so exit in themodified Epley manoeuvre) and the patient will be free of symptoms ('liberated'). If the nystagmus does not beat upward toward the affected ear, the clot is probably still inside the canal; if the nystagmus beats downward toward the unaffected ear (Fig. 4), the clot must have moved toward the cupula, causing an ampullopetal deflection. In either situation, the procedure should be repeated. If the nystagmus fails to beat upward following the second procedureand the BPPV persists, we schedule a return visit for the same manoeuvre. If the second session fails, we try a different liberatory manoeuvre (i.e. modified Epley, if wefirst used Semont, or viceversa). If both liberatory manoeuvresfail, weprescribeBrandt-D aroff exercises.

\section{NATURAL COURSE}

Thenatural history of BPPV is considered benign because it resolves spontaneously within weeks or months in most patients. H owever, in about $20-30 \%$ of thepatientsthecondition persists when untreated, and it recurs in another 30\% after variable periods for years.

\section{SURGICAL PROCEDURES}

In thoserarepatientswho do not respond even to appropriateand prolonged physical therapy, surgical plugging of the posterior semicircular canal via a transmastoid approach or surgical transection of the posterior ampullary nerve via a middleear approach can beconsidered.

In our experiencewith morethan 1000 patients with typical BPPV, very few failed to respond to physical therapy, and ultimately required selective surgical transection or canal plugging. We believe that surgical intervention is still too frequently performed, before all possibilities of physical therapy are completely exhausted. Thisview is shared by Epley (1995), who has invented his own effective liberatory procedure. Hebelieves that thedisability ensuing from multiple, unpredictable recurrencesisover thelongterm a morecommon indication for surgery.

\section{ACKNOWLEDGEMENTS}

I would like to thank M s J. Benson for copyediting the manuscript.

\section{REFERENCES}

Baloh RW, Hornrubia V \& Jacobson K (1987) Benign positional vertigo. Neurology, 37, 371-8.

Bárány R (1921) Diagnose von Krankheitserscheinungen im Bereiche des Otolithenapparates. Acta Otolaryngologica (Stockh), 2, 334-437.

Brandt Th (1999) Vertigo: its M ultisensory Syndromes, 2nd edn. Springer, London.

Brandt Th \& Daroff RB (1980) Physical therapy for benign paroxysmal positional vertigo. Archives of Otolaryngology, 106, 484-5.

BrandtTh \& SteddinS(1993) Current view of themechanism of benign paroxysmal positioning vertigo: Cupulolithiasis or canal olithiasis? Journal of Vestibular Research, 3, 373-82.

Brandt Th, Steddin S \& Daroff RB (1994) Therapy for benign paroxysmal positioning vertigo, revisited. Neurology, 44, 796-800.

Epley JM (1992) The canalith repositioning procedure: for treatment of benign paroxysmal positional vertigo. Otolaryngological H ead N eck Surgery, 107, 399-404.

EpleyJM (1995) Positional vertigo related to semicircular canalithiasis. Otolaryngological Head Neck Surgery, 112, 154-61.

Harvey SA, H ain TC \& Adamiec LC (1994) M odified liberatory maneuver: effective treatment for benign paroxysmal positional vertigo. Laryngoscope, 104, 1206-12.

Herdman SJ, Tusa RJ, Zee DS, Proctor LR \& M attox DE (1993) Single treatment approaches to benign paroxysmal positional vertigo. Archives of Otolaryngological H ead N eck Surgery, 119, 450-4.

SemontA, Freyss G \& VitteE (1988) Curing theBPPV with a liberatory maneuver. Advances in Otorhinolaryngology, 42, 290-3.

Serafini G, Palmierei AM R \& Simoncelli C (1996) Benign paroxysmal positional vertigo of posterior semicircular canal: results in 160 cases treated with Semont's maneuver. Annals of Otological Rhinological Laryngology, 105, 770-5.

Troost BT \& Patton JM (1992) Exercisetherapy for positional vertigo. Neurology, 42, 1441-4.
Fig. 4 Schematic drawing of an ineffective liberatory maneuver to be compared with Fig. 2, panel 3. After the patient is tilted to the right, the clot migrates back toward the cupula. Endolymph flow causes an ampullopetal cupula deflection with the nystagmus beating downward toward the unaffected ear. This indicates that the liberatory manouevre has failed. (From Brandt et al. 1994.) 